\title{
Muon Colliders and Neutrino Effective Doses
}

\author{
Joseph John Bevelacqua \\ Bevelacqua Resources
}

USA

\section{Introduction}

Lepton accelerators incorporate electron, muon, and tau beams. First generation lepton machines, electron accelerators, are basic research tools and their radiation characteristics are well established. A second generation muon machine presents additional research possibilities as well as new health physics challenges. Third generation tau accelerators are currently theoretical abstractions and little development has been forthcoming. Although this chapter focuses on muon colliders and their unique radiation characteristics, initial scoping calculatons for tau colliders are presented.

Neutrinos are electrically neutral particles, interact solely through the weak interaction, and have very small interaction cross sections (Particle Data Group 2010). They are present in the natural radiation environment due to cosmic rays, solar and terrestrial sources, and are produced during fission reactor and accelerator operations. From a health physics perspective these neutrino sources produce effective doses that are inconsequential. Although this will remain true for a number of years, planned muon accelerators or colliders will produce copious quantities of $\mathrm{TeV}$ energy neutrinos. In the $\mathrm{TeV}$ energy region, the health physics consequences of neutrinos can no longer be ignored. Upon operation of these accelerators, neutrino detection and the determination of neutrino effective doses will no longer be academic exercises, but will become practical health physics issues.

In a muon collider, neutrinos are produced when muons decay. The neutrino effective dose arises from neutrino interactions that produce showers or cascades of particles (e.g., neutrons, protons, pions, and muons). It is the particle showers that produce the dominant contribution to the neutrino effective dose (Bevelacqua, 2004).

Concerns for consequential neutrino effective doses have been previously postulated. Collar (1996) presented a hypothesis that the final stages of stellar collapse could produce neutrino effective doses that are sufficiently large to lead to the extinction of some species on earth. This concern has been challenged (Cossairt et al., 1997; Cossairt \& Marshall, 1997), but the potential concern for large neutrino effective doses, on the order of hundreds of $\mathrm{mSv} / \mathrm{y}$ or greater, remains, particularly for the planned muon colliders that will become operational in the next few decades of the 21st Century (Autin et al., 1999; Bevelacqua, 2004; Geer, 2010; King, 1999a; Kuno, 2009; and Zisman, 2011).

As background for muon colliders, an overview of the radiation environment at an electron accelerator is presented. This overview provides a foundation for a discussion of the characteristics of muon decays and the resultant neutrino effective doses. The characteristics 
of muon accelerators are addressed in this chapter and models for calculating the neutrino effective dose at a muon collider are provided. The radiological impacts of muon colliders and how basic dose reduction principles are affected by the underlying physics inherent in weak interaction processes are also discussed. Finally, a brief discussion of the neutrino effective doses anticipated at a third generation tau collider are provided.

\section{Electron-positron colliders}

Although this chapter addresses the neutrino effective dose from a muon collider, it is illustrative to provide a summary of the effects of other radiation types within a lepton collider (Bevelacqua, 2008, 2009, 2010a). These radiation fields are illustrated by considering an electron-positron collider. The radiation field within the muon collider facility are similar to those described in this section for electron-positron colliders.

An electron-positron collider accelerates electrons and positrons in circular rings before colliding the individual beams. There are a number of electron-positron colliders that have operated, are currently operating, or are being planned. These include the Large Electron Positron (LEP) Collider, and other machines summarized in the Review of Particle Properties (Particle Data Group, 2010). A new electron-positron machine, the International Linear Collider, is under design and is addressed from a health physics perspective in Bevelacqua (2008).

From an experimental physics perspective, electron-positron colliders have a number of advantages when compared to hadron colliders. First the collision results are less complex in terms of the particles produced, because electrons and positrons are fundamental particles without underlying structure or features. Hadrons are composed of quarks, but the electron and positrons have no such substructures. Therefore, the lepton's final state interactions are less complex than the structures that are produced from the interaction of the hadron's quarks. Particle interaction complexity is not the only advantage of electronpositron colliders.

The lepton colliders are also capable of achieving larger luminosities than hadron colliders. In addition, an order of magnitude less energy is required in electron-positron machines vice hadron colliders to achieve similar experimental results. For example, an electronpositron collider with a center-of-mass energy of $2 \mathrm{TeV}$ is roughly equivalent to a $20 \mathrm{TeV}$ center-of-mass energy hadron collider. In spite of these advantages, electron-positron collider health physics concerns exist (Bevelacqua; 2008, 2009, 2010a).

Electron-positron colliders produce more bremsstrahlung than hadron colliders. This bremsstrahlung production serves to limit the upper energies achieved by circular electronpositron colliders. In addition, electric power requirements rapidly increase with increasing energy unless beam power recovery mechanisms are developed and implemented.

The bremsstrahlung produced in a circular electron-positron collider is a fundamental concern that can only be decreased by increasing the circumference of the machine. The logical conclusion is to use an accelerator with an infinite radius (i.e., a linear collider). This is most easily achieved by replacing the dual beams in a circular collider with colliding beams from two linear colliders. 
The electron and positron beams produce a variety of radiation types that are derived from the direct beam and its interactions. Secondary radiation is produced from bremsstrahlung when beam particles strike accelerator components and from synchrotron radiation when beam particles are defected by magnetic fields.

Bremsstrahlung has a number of health physics consequences. These health physics issues include (NCRP 144, 2003): (1) electromagnetic cascade radiation containing high-energy photons, electrons, and positrons, (2) high-energy radiation including neutrons, pions, muons, and other hadrons, (3) activation of accelerator structures and components, (4) activation of air, cooling water, and soil, and (5) ozone and oxides of nitrogen produced in the air. Synchrotron radiation also has health physics consequences including: (1) electromagnetic cascade radiation, (2) photons, (3) neutrons, (4) activation of accelerator structures and components, (5) activation of air, cooling water, and soil, and (6) ozone and oxides of nitrogen produced in the air. These secondary radiation categories and their health physics consequences are addressed in more detail in subsequent discussion and in Bevelacqua $(2008,2009,2010 a)$.

The primary electron (positron) beams are contained within beam tubes, and secondary radiation is produced when the primary particles exit the beam tube either by design or accident. When electrons (positrons) exit the beam tube they strike accelerator components such as the beam tube structure, vacuum components, collimators, or structural members. When this occurs, the beam particle decelerates and radiates photons through the process of bremsstrahlung. The high-energy, bremsstrahlung photons produce electron-positron pairs that lead to additional bremsstrahlung. This process repeats itself, and produces an electromagnetic shower or cascade that contains numerous particles and a spectrum of photons having energies up to the kinetic energy of the initial beam particles.

A second category of secondary radiation occurs when the beam particles traverse the accelerator's magnetic fields. The magnetic field produces a force that alters the particle's trajectory. It also changes the particle's velocity and leads to the emission of photon radiation. This process is known as synchrotron radiation. Synchrotron radiation is related to bremsstrahlung because a change in velocity or acceleration is involved in both processes. However, the synchrotron radiation differs from the bremsstrahlung spectrum.

With bremsstrahlung, the photon energy extends from zero up to the energy of the beam particle. However, synchrotron radiation is governed by the configuration and strength of the magnetic field. Therefore, the synchrotron spectrum is machine specific. For example, CERN's decommissioned Large Electron-Positron collider had a synchrotron spectrum that extended from the range of visible light to a maximum intensity that occurred in the range of a few hundred $\mathrm{keV}$ (Bevelacqua, 2008). The synchrotron radiation intensity rapidly decreases from its peak value as the photon energy increases above a few $\mathrm{MeV}$. Both bremsstrahlung and synchrotron radiation induce an electromagnetic cascade.

The net result of the electromagnetic cascade is the deposition of energy in materials that are penetrated. This energy includes both particles stopped in the material and photon absorption. The photons produce additional secondary radiation and particles (e.g., photoneutrons) that activate accelerator materials. These same mechanisms lead to effective doses when personnel are in the presence of this radiation. These secondary radiation types 
are usually attenuated to insignificant levels by the concrete and earth shielding outside the accelerator tunnels containing the beam tubes.

From a health physics perspective, the energy loss of the circulating, accelerating electrons and positrons produces synchrotron radiation (photons). Given the mass of the electrons and positrons, their trajectories are easily altered. Therefore, synchrotron radiation is expected to be a large fraction of the available beam power. The synchrotron radiation requires shielding, and the extent of the shielding depends on the specific location within the accelerator facility.

The amount of synchrotron radiation depends on the specific design characteristics of the electron-positron collider. Dominant factors governing the production of synchrotron radiation are the beam power and radius of curvature of the accelerator ring. From a practical standpoint, radiation generated from the circulating electron and positron beams occurs within the unoccupied shielded ring and is not normally a health physics issue.

The dominant contributors to the radiation environment at an electron-positron facility include electromagnetic cascade showers, external bremsstrahlung, photoneutrons, muons, and synchrotron radiation. Muon pair production in the Coulomb field of a nucleus is possible above a photon energy of about $211 \mathrm{MeV}$. This process is analogous to electronpositron pair production, but the muon pair production cross-sections are smaller by a factor of about 40,000 due to the differences in electron $(0.511 \mathrm{MeV})$ and muon $(105.7 \mathrm{MeV})$ masses (Bevelacqua, 2008).

The dominant muon pair production process is coherent muon production. In coherent production, the target nucleus remains intact as it recoils from the photon interaction. In a few percent of the time, the nucleus breaks-up with the resultant emission of muons. Muons also result from the decay of photopions and photokaons. However, the number of muon decays in a conventional electron-positron collider is not sufficient to produce a neutrino effective dose concern. To understand the neutrino effective dose from a muon collider, it is necessary to understand neutrino physics and neutrino interactions.

\section{Basic neutrino physics}

The current view of elementary particle physics is embodied in the Standard Model of Particle Physics (Cottingham \& Greenwood, 2007; and Griffiths, 2008) that assumes all matter is composed of three types of fundamental or elementary particles: leptons, quarks, and mediators of the fundamental interactions. Bevelacqua (2010b) provides a description of the Standard Model from a health physics perspective.

Leptons interact primarily through the weak interaction and electrically charged leptons also experience the effects of the electromagnetic force. They are not affected by the strong interaction. The leptons may be naturally grouped into three families or generations as $\left(e^{-}, v_{e}\right),\left(\mu^{-}, v_{\mu}\right)$, and $\left(\tau^{-}, v_{\tau}\right)$.

Neutrinos are neutral leptons, once believed to be massless, but now evidence suggests they have a non-zero mass (Particle Data Group, 2010). The electron and muon neutrinos are well studied, but less is known about tau neutrinos 
To allow for massive neutrinos, the Standard Model must be modified and its assumptions altered. However, current experimental knowledge of neutrino properties does not permit the selection of a specific modification to the model. For example, it is not known if neutrino masses are to be interpreted as evidence of new, light, fermionic degrees of freedom (e.g., Dirac neutrinos), new, heavy, degrees of freedom (e.g., Majorana neutrinos), or whether a more complicated electroweak-symmetry-breaking interaction is present. However, the Standard Model is sufficient for the purposes of this chapter.

Within the Standard Model, neutrino effective doses are determined from the muon decay processes:

$$
\begin{aligned}
& \mu^{-} \rightarrow e^{-}+v_{\mu}+\bar{v}_{e} \\
& \mu^{+} \rightarrow e^{+}+\bar{v}_{\mu}+v_{e}
\end{aligned}
$$

The neutrino effective doses depend on the number of muon decays, and the subsequent production of neutrinos. Specific effective dose relationships are provided in subsequent discussion.

\section{Neutrino interactions related to effective dose}

In a muon collider, muon decays arise principally from Eqs. 1 and 2 that produce neutrinos and antineutrinos. The neutrinos interact through a variety of complex processes. A neutrino interaction discussion is simplified by following the methodology of Cossairt et al. (1997) and defining four processes (A, B, C, and D) to describe neutrino interactions with matter. The deposition of energy into tissue defines the effective dose (Bevelacqua, 2009, 2010a).

Process A involves neutrino scattering from atomic electrons. Electrons that recoil from elastic neutrino scattering deposit their energy in tissue and produce a neutrino effective dose. Process A occurs over a wide range of energy and the electron tissue interaction may involve multiple scattering of electrons.

In Process B, neutrinos interact coherently with nuclei. This process is only effective for low neutrino energies where the neutrino wavelength is too long to resolve the individual nucleons within the nucleus. At higher energies, Processes $C$ and $D$ become more important. Process $B$ leads to low-energy ions having large linear energy transfer values. These ions deposit their energy into tissue according to their ranges, which are typically $\ll 1 \mathrm{~cm}$. Although Process B is independent of the neutrino generation, the cross section for neutrinos is about twice the antineutrino cross section (King 1999a).

Process $\mathrm{C}$ involves neutrino scattering from nucleons without shielding between the neutrinos and tissue. At energies below about $500 \mathrm{MeV}$, tissue dose is due to recoil nucleons. As the neutrino energy increases above about $0.5 \mathrm{GeV}$, secondary particle production increases. Eventually, these secondary particles produce particle showers or cascades in tissue. Process $C$ is independent of the neutrino generation, affecting all three generations in the same manner.

Process D is similar to Process $C$ with the exception that the neutrinos are shielded before striking tissue. Neutrinos with energy greater than about $0.5 \mathrm{GeV}$, emerging from a layer of 
material (e.g., earth shielding), result in a larger effective dose than unshielded neutrinos. The increase in effective dose arises from the fact that the tissue is exposed to the secondary particles produced by neutrino interactions in the shielding material as well as the neutrino beam. Process $\mathrm{D}$ is also independent of the neutrino generation.

A process that involves an increase in effective dose with added shielding is unique. One of the basic tenants for reducing effective dose for most radiation types (e.g., alpha and beta particles, heavy ions, muons, neutrons, photons, pions, and protons) is shielding the radiation source (Bevelacqua, 2009 and 2010a). The unique nature of Process D has a significant impact on the evaluation and control of neutrino effective dose.

\section{Neutrino beam characteristics at a muon collider}

Neutrinos are produced when the muon beam particles decay (See Eqs. 1 and 2). Weak interactions of muon neutrinos can be described in terms of two broad categories: charged current and weak current interactions. Charged current interactions involve the exchange of W-bosons to form secondary muons. Neutral current interactions produce uncharged particles through the exchange of Z-bosons. Both types of interactions produce hadron particle showers. Therefore, the neutrino induced radiation hazard will include secondary muons and hadronic showers. The hadronic showers have a much shorter range than the muons, but the number of particles in a hadronic shower can be quite large. The neutrino radiation hazard arises from these penetrating charged particle showers (Bevelacqua, 2008).

For TeV energy neutrinos, direct neutrino interactions in man account for less than $1 \%$ of the total effective dose because the primary hadrons from the neutrino interactions will typically exit the person before producing a charged particle shower (King, 1999b; Cossairt et al., 1996, 1997). Most of the neutrino effective dose is derived from particle showers produced in the shielding material.

The muon beam and subsequent neutrino beam are assumed to be well-collimated and to have a minimum divergence angle. For practical situations, the muons in the accelerator beam will have a small divergence angle and will be periodically focused using electromagnetic fields to ensure their collimation. No beam divergence is assumed in the subsequent calculations. Therefore, the actual beam will be somewhat more diffuse than assumed in the neutrino effective dose calculations. The neutrino beam will still produce particle showers, but they will be somewhat broader and less intense than the assumed well-collimated result. The beam divergence is analogous to the divergence of a laser beam as it exits an aperture (Bevelacqua, 2009, 2020).

The magnitude of the effective dose from a particle shower is dependent on the material in the interaction region lying directly upstream of the individual being irradiated. Calculation of the neutrino effective dose considers the configuration where a person is (1) completely bathed in the neutrino beam, and (2) is surrounded by material that will produce particle showers from neutrino interactions. These requirements lead to a bounding set of effective dose predictions.

These assumptions are too conservative for the $\mathrm{TeV}$ energies that will be encountered in mature muon colliders, but they provide a bounding neutrino effective dose result given the current level of design. Basic physics principles suggest that the neutrino interactions will be 
more peaked in the beam direction as the muon energies increase. In addition, the neutrino beam radius ( $\mathrm{r}$ ) will be relatively small and is given by (King, 1999b):

$$
\mathrm{r}=\theta \mathrm{L}
$$

where $\theta$ is called the characteristic angle, opening half-angle, or half-divergence angle of the muon decay cone

$$
\theta=\frac{m c^{2}}{E}
$$

In Eqs. 3 and 4, $\mathrm{L}$ is the distance to the point of interest such as the distance from the muon decay location to the earth's surface, $\theta$ is given in radians, $\mathrm{E}$ is the muon beam energy, and $m c^{2}$ is the rest mass of the muon $(105.7 \mathrm{MeV})$. As the muon energy increases, the neutrino beam radius and size of the resultant hadronic showers tend to be smaller than the size of a person.

The characteristic angle varies inversely with energy. If $\mathrm{E}$ is expressed in $\mathrm{TeV}$ :

$$
\theta \approx \frac{10^{-4}}{E[\mathrm{TeV}]}
$$

Therefore, the emergent neutrino beam will consist of a narrow diverging beam that is conical in shape.

Table 1 summarizes straw-man muon collider parameters (King, 1999b). It should be noted that the straw-man muon colliders are constructed below the earth's surface to provide muon shielding. However, the neutrino attenuation length is too long for the beam to be appreciably attenuated by any practical amount of shielding, including the expanse of ground between the collider and its exit from the surface of the earth. Therefore, the effective dose reduction principle as applied to neutrinos will no longer include shielding as an element. In fact, shielding the neutrino beam will produce hadronic showers and increase the effective dose. This peculiar behavior has its basis in the nature of the weak interaction, the uncharged nature of the neutrino, and the $\mathrm{TeV}$ energies that will be encountered in proposed muon colliders.

\begin{tabular}{|c|c|c|c|}
\hline $\mathrm{E}(\mathrm{TeV})$ & 2 & 5 & 50 \\
\hline $\mathrm{L}(\mathrm{km})$ & 62 & 36 & 36 \\
\hline $\mathrm{r}(\mathrm{m})$ & 3.3 & 0.8 & 0.08 \\
\hline Collider depth $(\mathrm{m})$ & 300 & 100 & 100 \\
\hline
\end{tabular}

Table 1. Straw-Man Muon Collider Parameters.

The neutrinos exiting a muon collider will not only have a narrow conical shape, but will also have an extent that is quite long. The long, narrow plume of neutrinos will produce secondary muons and hadronic showers at a significant distance from the muon collider. This distance will be greater than tens of kilometers for $\mathrm{TeV}$ muon energies. 


\section{Neutrino interaction model}

Neutrinos can interact directly with tissue or with intervening matter to produce charged particles that result in a biological detriment. The radiation environment is complex and simulations (e.g., Monte Carlo methods) can be used to model the dynamics of the neutrino interaction including the energy and angular dependence of each particle (e.g., $v_{e}, \bar{v}_{e}, v_{\mu}$, $\bar{v}_{\mu}, v_{\tau}, \bar{v}_{\tau}, \mathrm{e}, \mu, \tau$, and hadrons) involved in the interaction. Performing a neutrino simulation is too dependent on specific accelerator characteristics and will not add to the health physics presentation. Rather than performing a Monte Carlo simulation, we follow the analytical approach of Cossairt et al. (1997) and King (1999b) to quantify the neutrino effective dose. This approach is acceptable in view of the current uncertainties in muon collider technology and the nature of the neutrino interaction for both charged current (CC) and neutral current (NC) weak processes (King, 1999c).

Following King (1999c), the dominant interaction of TeV-scale neutrinos is deep inelastic scattering with nucleons that include $\mathrm{CC}$ and NC components. In the NC process, the neutrino is scattered by a nucleon $(\mathrm{N})$ and loses energy with the production of hadrons $(\mathrm{X})$ through a $v+N \rightarrow v+X$ reaction. This $\mathrm{NC}$ reaction contributes about 25 percent of the total cross section. This NC process can be interpreted as elastic scattering off one of the quarks (q) inside the nucleon through the exchange of a virtual $\mathrm{Z}^{0}$ boson $(v+q \rightarrow v+q)$.

CC scattering is similar to NC scattering except that the neutrino is converted into its corresponding charged lepton (l). This includes reactions such as $v+N \rightarrow l^{-}+X$ and $\bar{v}+N \rightarrow l^{+}+X$ where 1 is an electron/muon for electron/muon neutrinos. At the quark level, a charged $\mathrm{W}$ boson is exchanged with a quark to produce another quark ( $\left.\mathrm{q}^{\prime}\right)$ whose charge differs by one unit through processes such as $v+q \rightarrow l^{-}+q^{\prime}$ and $\bar{v}+q^{\prime} \rightarrow l^{+}+q$.

The final state quarks produce hadrons on a nuclear distance scale that contribute to the effective dose. The CC and NC scattering processes are included in the Process A -D descriptions noted in previous discussion.

\section{Neutrino effective dose}

A muon collider provides a platform for colliding beams of muons $\left(\mu^{-}\right)$and antimuons $\left(\mu^{+}\right)$(Geer, 2010). The collider may involve a pair of linear accelerators with intersecting beams or a storage ring that circulates the muons and antimuons in opposite directions prior to colliding the two beams. The accelerator facility energy is usually expressed as the sum of the muon and antimuon energies. For example, a $100 \mathrm{TeV}$ accelerator consists of a $50 \mathrm{TeV}$ muon beam and a $50 \mathrm{TeV}$ antimuon beam. Since muon colliders produce large muon currents, neutrinos will be copiously produced from the decay of both muons and antimuons (See Eqs. 1 and 2).

Neutrino effective dose calculations are performed for two potential muon collider configurations. The first configuration utilizes the intersection of the beams of two muon linear colliders. The linear collider effective dose model incorporates an explicit representation of the neutrino cross section and evaluates the effective dose assuming specific values for the muon energy, number of muon decays per year, and accelerator 
operational characteristics (e.g., accelerator gradient or the increase in muon energy per unit accelerator length). The operational parameter approach is more familiar to high-energy physicists, but it serves to illustrate the sensitivity of the neutrino effective dose to the key muon collider's operating parameters.

The second configuration is a circular muon collider. The neutrino effective dose for the circular muon collider involves an integral over energy of the differential fluence and fluence to dose conversion factor. This approach is more familiar to health physicists, but much of the muon collider's operating parameters are absorbed into other parameters and are not explicitly apparent. Using both approaches yields not only the desired neutrino effective dose, but also illustrates the sensitivity of the effective dose to a number of accelerator parameters and operational assumptions.

\subsection{Bounding neutrino effective dose - linear muon collider}

The bounding neutrino effective dose from a linear muon collider is derived following King (1999b) and is based on the effective dose from a straight section (ss) of a circular muon collider. This derivation incorporates a limiting condition from a circular accelerator with a number of straight sections as part of the facility. Parameters unique to the circular collider such as the ring circumference and straight section length appear in intermediate equations, but cancel in the final effective dose result. In the linear muon collider, the muon beam is assumed to be well-collimated.

In a linear muon collider, the total neutrino effective dose $(\mathrm{H})$ is defined in terms of an effective dose contribution $\delta \mathrm{H}(\mathrm{E})$ received in each energy interval $\mathrm{E}$ to $\mathrm{E}+\mathrm{dE}$ as the muons accelerate to the beam energy $\mathrm{E}_{\mathrm{o}}$ :

$$
H=\int_{0}^{E_{o}} d E \delta H(E)
$$

The effective dose contribution $\delta \mathrm{H}(\mathrm{E})$ is written as (King, 1999b):

$$
\delta H(E)=H^{\prime} \frac{1}{f_{s s}} \frac{d f(E)}{d E}
$$

where $\frac{d f(E)}{d E} d E$ is the fraction of muons that decay via Eqs. 1 and 2 in the energy interval $E$ to $\mathrm{E}+\mathrm{dE}$, which may be written as:

$$
\frac{d f(E)}{d E}=\frac{1}{\gamma \beta c \tau g}
$$

where

$$
\gamma=\frac{E_{o}}{m c^{2}}
$$


In Eq. $8, \beta=\mathrm{v} / \mathrm{c}, \mathrm{\tau}$ is the muon mean lifetime $\left(2.2 \times 10^{-6} \mathrm{~s}\right)$, and $\mathrm{g}$ is the accelerator gradient $(\mathrm{dE} / \mathrm{dl})$. The other parameters appearing in Eq. 7 include $\mathrm{f}_{\mathrm{ss}}$ (the ratio of the straight section length to the ring circumference) and $\mathrm{H}^{\prime}$ (the effective dose that is applicable as the muon energy reaches the $\mathrm{TeV}$ energy range), where

$$
f_{s s}=\frac{l_{s s}}{C}
$$

In Eq. 10, C is the ring circumference:

$$
C=\frac{2 \pi E_{0}}{0.3 \bar{B}}
$$

In Eqs. $9-11, v$ is the muon velocity, $1_{s s}$ is the straight section length, $E_{o}$ is the muon energy, $\bar{B}$ is the ring's average magnetic induction, and $N$ is the number of muon decays in a year.

In the narrow beam approximation, the effective dose is independent of distance $(\mathrm{L})$ for $\mathrm{L}<$ $5 \mathrm{E}_{\mathrm{o}}$ (King, 1999b) where $\mathrm{L}$ is expressed in $\mathrm{km}$ and $\mathrm{E}_{\mathrm{o}}$ in $\mathrm{TeV}$. Using this approximation,

$$
H^{\prime}=K^{\prime} N l_{s s} \bar{B} E X
$$

where $\mathrm{K}^{\prime}$ is a constant that depends on the units used to express the various quantities appearing in Eq. 12, and $X=X(E)$ is the cross section factor defined in subsequent discussion.

Combining these results leads to the annual neutrino effective dose $(\mathrm{H})$ in $\mathrm{mSv} / \mathrm{y}$ :

$$
H=\frac{N K}{g} \int_{0}^{E o} E X(E) d E
$$

where $\mathrm{K}=6.7 \times 10^{-21} \mathrm{mSv}-\mathrm{GeV} / \mathrm{m}-\mathrm{TeV}^{2}$ if $\mathrm{g}$ is expressed in $\mathrm{GeV} / \mathrm{m}, \mathrm{N}$ is expressed in muon decays per year, $\mathrm{E}$ is the muon energy in $\mathrm{TeV}$, and the cross section factor is dimensionless (Bevelacqua, 2004).

In deriving the linear muon collider effective dose relationship, a number of assumptions were made (Bevelacqua, 2004). These assumptions are explicitly listed to ensure the reader clearly understands the basis for Eq. 13. The relevant assumptions include applicability of the narrow beam approximation. The individual receiving the effective dose is assumed to be: (1) uniformly irradiated, (2) within the footprint of the neutrino beam, (3) within the footprint of the hadronic particle shower that results from the neutrino interactions, and (4) irradiated by only one of the linear muon accelerators whose energy is one-half the total linear muon collider energy. Given the TeV muon energies and the earth shielding present, charged particle equilibrium exists and Process D dominates the neutrino effective dose. In addition, the muon beam is well-collimated, the neutrino effective dose calculation assumes a $100 \%$ occupancy factor, and the neutrino effective dose is an annual average based on the number of muon decays in a year.

The cross section factor is a parameterization of the neutrino cross section (See Table 2) in terms of a logarithmic energy interpolation (Quigg, 1997). The numerical factors in the Table 
2 expressions $(1.453,1.323,1.029,0.512$, and 0.175$)$ are the total summed neutrino-nucleon and antineutrino-nucleon cross sections divided by energy at neutrino energies of $0.1,1,10$, 100 , and $1000 \mathrm{TeV}$, respectively, given in units of $10^{-38} \mathrm{~cm}^{2} / \mathrm{GeV}$. As an approximation, the muon energies in Table 2 are set equal to the corresponding neutrino energies. Following Quigg (1997), the cross section factor is a dimensionless number and is normalized such that $\mathrm{X}(\mathrm{E}=0.1 \mathrm{TeV})=1.0$.

\begin{tabular}{|c|c|}
\hline Muon Energy Range (TeV) & $X(E)$ \\
\hline $\mathrm{E}<1$ & $(-1.453 \alpha+1.323(\alpha+1)) / 1.453$ \\
\hline $1<\mathrm{E}<10$ & $(1.323(1-\alpha)+1.029 a) / 1.453$ \\
\hline $10<\mathrm{E}<100$ & $(1.029(2-a)+0.512(a-1)) / 1.453$ \\
\hline $100<\mathrm{E}<1,000$ & $(0.512(3-a)+0.175(a-2)) / 1.453$ \\
\hline $\mathrm{E}>1,000$ & $(0.175 / 1.453) 3^{3-\alpha}$ \\
\hline
\end{tabular}

Table 2. Cross Section Factor X(E) as a Function of Muon Energy.

Eq. 13 may be approximated by replacing the energy-weighted integral of $X(E)$ by its value at $\mathrm{E}=\mathrm{E}_{\mathrm{o}} / 2$. This choice is acceptable given the energy dependence of the cross section and the associated uncertainties in the collider design parameters. With this selection, the annual neutrino effective dose $(\mathrm{mSv} / \mathrm{y})$ becomes:

$$
H=\frac{K N}{2 g} \mathrm{X}\left(E_{o} / 2\right) E_{o}^{2}
$$

As a practical example (Zimmerman, 1999), consider a 1,000 TeV muon linear accelerator assuming $\mathrm{E}_{\mathrm{o}}=500 \mathrm{TeV}$ (i.e., two, $500 \mathrm{TeV}$ linear muon accelerators) and $\mathrm{N}=6.4 \times 10^{18}$ muon decays per year. Using these values in Eq. 14 with a $\mathrm{g}=1 \mathrm{GeV} / \mathrm{m}$ value leads to an annual effective neutrino dose of $1.4 \mathrm{~Sv} / \mathrm{y}$, which is a significant value that cannot be ignored. Health physicists at a linear muon collider will need to contend with large neutrino effective doses within and outside the facility. Table 3 provides expected annual neutrino effective doses for a variety of accelerator energies using the same $\mathrm{N}$ and $\mathrm{g}$ values noted above and the narrow beam approximation.

\begin{tabular}{|c|c|c|}
\hline $\begin{array}{c}\text { Accelerator Facility Energy } \\
(\mathrm{TeV})\end{array}$ & $\begin{array}{c}\text { Muon Beam Energy } \\
(\mathrm{TeV})\end{array}$ & $\begin{array}{c}\mathrm{H} \\
(\mathrm{mSv} / \mathrm{y})\end{array}$ \\
\hline 0.1 & 0.05 & $5.7 \times 10^{-5}$ \\
\hline 1 & 0.5 & $5.2 \times 10^{-3}$ \\
\hline 10 & 5 & 0.45 \\
\hline 100 & 50 & 30 \\
\hline 500 & 250 & 440 \\
\hline 1,000 & 500 & $1.4 \times 10^{3}$ \\
\hline 5,000 & 2,500 & $1.5 \times 10^{4}$ \\
\hline 10,000 & 5,000 & $4.2 \times 10^{4}$ \\
\hline 50,000 & 25,000 & $4.8 \times 10^{5}$ \\
\hline
\end{tabular}

Table 3. Annual Neutrino Effective Doses for a Linear Muon Collider Using the Narrow Beam Approximation. 
The values of Table 3 suggest that the annual effective dose limit for occupational exposures of $20 \mathrm{mSv} / \mathrm{y}$ and the annual effective dose limit to the public $(1 \mathrm{mSv} / \mathrm{y})$ can be exceeded by $\mathrm{TeV}$ energy muon accelerators (ICRP 103, 2007). The values in Table 3 also exceed the emergency effective dose limit of $250 \mathrm{mSv}$ set for the Fukushima Daiichi accident that is based on ICRP 60 (1991).

A TeV - PeV scale muon collider will also challenge the acute lethal radiation dose $\left(\mathrm{LD}_{50,30}\right)$ of about 4 Gy (Bevelacqua 2010a). Although the feasibility of $\mathrm{TeV}$ - PeV scale machines remains to be determined, the significant radiation hazards associated with their operation merits careful attention to the effects of neutrino effective doses at offsite locations.

Selecting an accelerator location will be an issue for $\mathrm{TeV}$ energy muon linear colliders due to public radiation concerns arising from neutrino interactions. Given these radiation concerns, a muon collider location may be restricted to low population or geographically isolated areas to minimize the public neutrino effective dose.

\subsection{Bounding neutrino effective dose - circular muon collider}

The bounding neutrino effective dose for a circular muon collider could be obtained using the methodology of the previous section. However, a number of operational assumptions including the ring circumference and average magnetic induction would be required. Instead, we use an alternative approach to illustrate the various methods than can be utilized to determine the neutrino effective dose as a function of distance. To accomplish this, consider the energy distribution or differential fluence $d N_{i}\left(E_{i}\right) / d E_{i}$ where $\mathrm{N}_{\mathrm{i}}$ is the number of neutrinos of generation $i$ per unit area, $E_{i}$ is the neutrino energy, and $i=1,2$, and 3 for the three neutrino generations. The neutrino effective dose $\mathrm{H}$ can be determined once the neutrino fluence to effective dose conversion factor $C\left(E_{i}\right)$ is known.

Cossairt et al. (1997) provide an approach for treating the neutrinos and their antiparticles in the first two generations. In view of the limited data, Cossairt et al. (1997) did not consider the generation 3 neutrinos, but these neutrinos become more important as the accelerator energy increases.

One of the initial goals of a muon accelerator will be the development of a pure muon neutrino beam to investigate the magnitude of the neutrino mass. Focusing on the muon neutrino is also warranted because Cossairt et al. (1997) provides a muon neutrino fluence to effective dose conversion factor. Following Cossairt et al. (1997) and Silari \& Vincke (2002), we limit the subsequent discussion to muon neutrinos that result from muon decays (Eq. 1) in a circular muon collider and drop the subscript $\mathrm{i}$ :

$$
H=\int_{0}^{E_{o}} \frac{d N(E)}{d E} C(E) d E
$$

where $E_{o}$ is the energy of the primary muons before decay.

Silari \& Vincke (2002) provide a differential fluence value in the laboratory system that is averaged over all neutrino production angles. They also assume the accelerator's shielding is thick enough to attenuate the primary muon beam, and that it is thicker than the range of 
all secondary radiation. Accordingly, the neutrino radiation is in equilibrium with its secondary radiation.

Using the equilibrium condition and averaging over all production angles, provides the following differential fluence relationship for the neutrino radiation from a circular muon collider (Silari \& Vincke, 2002):

$$
\frac{d N(E)}{d E}=\frac{2}{E_{o}}\left(1-\frac{E}{E_{o}}\right) \Phi
$$

where $N(E)$ is the number of neutrinos per unit area, $E$ is the neutrino energy, $E_{o}$ is the energy of the primary muons before decay, and $\Phi$ is the integral neutrino fluence (total number of neutrinos per unit area) following the muon decays.

For secondary particle equilibrium, the fluence to effective dose conversion factor relationship of Cossairt et al. (1997) is used:

$$
C(E)=K E^{2}
$$

Eq. 17 was derived for the neutrino energy range of $0.5 \mathrm{GeV}$ to $10 \mathrm{TeV}$. In deriving the muon neutrino effective dose to fluence conversion factor of Eq. 17, Cossairt et al. (1997) did not consider the effects of the third lepton generation.

In Eq. $17, \mathrm{~K}=10^{-15} \mu \mathrm{Sv}-\mathrm{cm}^{2} / \mathrm{GeV}^{2}$. In view of the trend in the neutrino data (Particle Data Group, 2010; Quigg, 1997), Eq. 17 is used at energies beyond those considered by Cossairt et al. (1997). This is reasonable because increasing energy and increasing number of secondary shower particles (hadrons) is the main reason for the rising fluence to effective dose conversion factor with increasing neutrino energy for the equilibrium (shielded neutrino) case or process $\mathrm{D}$ described earlier. It is also reasonable because the neutrino attenuation length $(\lambda)$ decreases with increasing energy of the primary neutrinos. Although TeV energy units are used in the final result, $\mathrm{GeV}$ units are used in the derivation of the neutrino effective dose to facilitate comparison with Silari \& Vincke (2002) and Johnson et al. (1998). Prior to developing the neutrino effective dose relationship for a circular muon collider, the neutrino attenuation length is briefly examined.

The neutrino attenuation length is written in terms of the neutrino interaction cross section $\sigma_{\mathrm{v}}$ :

$$
\lambda=\frac{A}{\rho N_{A} \sigma_{v}}=\frac{1}{N \sigma_{v}}
$$

where $A$ and $\rho$ are the atomic number and density of the shielding medium, $N_{A}$ is Avogadro's number, $\mathrm{N}$ is the number density of atoms of the shielding medium per unit volume, and $\sigma_{\mathrm{v}}$ is on the order of $10^{-35} \mathrm{~cm}^{2}(\mathrm{E} / 1 \mathrm{TeV}$ ) (Johnson et al. ,1998) where the neutrino energy is expressed in $\mathrm{TeV}$.

These results permit the neutrino attenuation length to be written as (Johnson et al. ,1998):

$$
\lambda=0.5 \times 10^{6} \mathrm{~km}\left(\frac{1 \mathrm{TeV}}{E}\right)\left(\frac{3 \mathrm{~g} / \mathrm{cm}^{3}}{\rho}\right)
$$


Since the neutrino attenuation length is very long, the neutrino fluence is very weakly attenuated while traversing a shield. Therefore, shielding is not an effective dose reduction tool for neutrinos.

The effective dose arising from an energy independent neutrino fluence spectrum is accomplished by performing the integration of Eq. 15 using Eqs. 16 and 17:

$$
H=\int_{0}^{E_{o}} \frac{2}{E_{o}}\left(1-\frac{E}{E_{o}}\right) \Phi\left(K E^{2}\right) d E=\frac{K}{6} E_{o}^{2} \Phi
$$

where $\mathrm{H}$ is the annual neutrino effective dose in $\mu \mathrm{Sv}$ and $\Phi$ is the total number of neutrinos per unit area that is assumed to be independent of energy (Johnson et al. ,1998).

The neutrino fluence $\Phi$ is the total number of neutrinos traversing a surface behind the shielding. The surface is governed by the divergence of the neutrino beam and the distance $r$ from the neutrino source. The neutrino's half-divergence angle $(\theta)$ is:

$$
\theta=\frac{m c^{2}}{E}=\frac{1}{\gamma} \approx \frac{1}{10 E_{o}}
$$

where $\mathrm{mc}^{2}$ is the muon rest mass in $\mathrm{MeV}, \mathrm{E}$ is the muon energy, $\theta$ is the opening half-angle or characteristic angle of the decay cone expressed in radians, and $E_{o}$ is the energy of the primary muon beam in $\mathrm{GeV}$.

The neutrino fluence $\Phi$ at a given distance $r$ from the muon decay point is just the number of neutrinos $\mathrm{N}$ per unit area:

$$
\Phi=\frac{N}{\pi(\theta r)^{2}}
$$

Combining Eqs. 20 - 22 and using the numerical value for $\mathrm{K}$ yields a compact form for the annual neutrino effective dose from a circular muon collider:

$$
H=\frac{10^{-15} E_{o}^{2}}{6} \frac{N}{\pi(\theta r)^{2}}=\frac{10^{-15} E_{o}^{2}}{6} \frac{N}{\pi} \frac{\left(10 E_{o}\right)^{2}}{r^{2}}=\frac{10^{-13} E_{o}^{4} N}{6 \pi r^{2}} \frac{\mu S v-c m^{2}}{G e V^{4}}
$$

The circular muon collider neutrino effective dose of Eq. 23 has a very strong dependence on the neutrino energy.

Eq. 23 provides the neutrino effective dose assuming all muons decay at the same point. Recognizing that the muons can decay at all storage ring locations with equal probability provides a more physical description of the effective dose. For facilities such as the European Laboratory for Particle Physics (CERN), the neutrino effective dose may to be calculated as an integral over the length of the return arm (1) (Silari \& Vincke, 2002) of the storage ring pointing toward the surface from $d$ to $d+1$, where $d$ is the thickness of material traversed by the neutrino beam between the end of the return arm and the surface of the earth along the direction of the return arm. The quantity $d$ may also be described as the approximate minimum thickness of earth needed to absorb the circulating muons if beam misdirection or total beam loss occurs (i.e., the beam exits the 
facility). Recognizing that the muons may decay at any location along the return arm, leads to the neutrino effective dose:

$$
H=\frac{10^{-13} E_{o}^{4}}{6 \pi} \int_{d}^{d+l} \frac{N}{l} \frac{d r}{r^{2}}=\frac{10^{-13} E_{o}^{4} N}{6 \pi l}\left(\frac{1}{d}-\frac{1}{d+l}\right) \frac{\mu S v-c m^{2}}{G e V^{4}}
$$

Silari \& Vincke (2002) provides parameters for the planned muon facility at CERN. For a 50 $\mathrm{GeV}$ muon energy in the storage ring, $\mathrm{N}=10^{21}$ muons per year decaying in the ring, a return arm length pointing toward the surface $\left(1=6.0 \times 10^{4} \mathrm{~cm}\right)$, and a $100 \mathrm{~m}$ thickness of material (d) traversed by the neutrino beam between the end of the return arm and the surface, a surface neutrino effective dose of $47 \mathrm{mSv} / \mathrm{yr}$ is predicted. Since the planned CERN design has 3 return arms, the effective dose rate at the end of one of the arms would be about 16 $\mathrm{mSv} / \mathrm{y}(47 \mathrm{mSv} / 3)$. Increasing muon energy will lead to higher muon effective dose rates, additional muon shielding requirements, and will force the collider deeper underground (See Table 4, derived from Silari \& Vincke, (2002).

\begin{tabular}{|c|c|c|c|c|}
\hline Muon Energy $(\mathrm{TeV})$ & $\mathrm{d}(\mathrm{m})$ & $\mathrm{L}(\mathrm{km})$ & $\varphi(\mathrm{mrad})$ & $\theta(\mu \mathrm{rad})$ \\
\hline 1 & 100 & 36 & 5.6 & 106 \\
\hline 2 & 100 & 36 & 5.6 & 53 \\
\hline 5 & 200 & 51 & 8 & 21 \\
\hline 10 & 500 & 80.5 & 12.5 & 11 \\
\hline
\end{tabular}

Table 4. Geometrical Parameters for Representative Cases of Circular Muon Colliders

These results suggest that the circular muon collider be installed underground to shield the muon beam in the event the beam becomes misdirected. This required shielding is determined by the muon energy loss (Silari \& Vincke, 2002):

$$
\frac{d E}{d x}=0.6 \frac{\mathrm{TeV}}{\mathrm{km}}\left(\frac{\rho}{3 \mathrm{~g} / \mathrm{cm}^{3}}\right)
$$

When compared to muons, neutrinos have a much smaller interaction cross section. The earth shielding that completely attenuates the muons will have a negligible effect on the neutrinos. Accordingly, the neutrinos will produce a nontrivial annual effective dose at the earth's surface where the beam emerges. In order to evaluate the magnitude of this neutrino effective dose, assume the earth is a sphere, and a horizontal, circular muon collider is situated a depth $\mathrm{d}$ below the earth's surface. The neutrino beam exit point from the earth will be at a horizontal distance L given by Silari \& Vincke (2002):

$$
L=\sqrt{2 d R-d^{2}} \approx \sqrt{2 d R} \approx 36 \mathrm{~km} \sqrt{\frac{d}{100 m}}
$$

where $R=6400 \mathrm{~km}$ is the earth's radius. Table 4 provides representative values of $\mathrm{d}$ and $\mathrm{L}$.

In addition to $\mathrm{d}$ and $\mathrm{L}$, a number of other relevant parameters associated with the circular collider of Eq. 26 are summarized in Table 4. In Table 4, $\varphi$ is the half-angle subtended by the horizontal accelerator beam with respect to the earth's center before it exits the earth: 


$$
\operatorname{Sin} \varphi=\mathrm{L} / \mathrm{R}
$$

The functional form of Eq. 24 suggests that the calculation of neutrino effective dose from a circular muon collider is dependent of the assumed physical configuration and beam characteristics. An estimate of the neutrino effective dose for a circular muon collider can be made using Eq. 23. For comparison with Eq. 14, Eq. 23 is rewritten in terms of $\mathrm{TeV}$ and $\mathrm{mSv}$ units:

$$
H=\frac{10^{-4} E_{o}^{4} N}{6 \pi r^{2}} \frac{m S v-c m^{2}}{T e V^{4}}
$$

where $\mathrm{N}$ is the number of muon decays per year, $\mathrm{E}_{\mathrm{o}}$ is the muon energy in $\mathrm{TeV}, \mathrm{r}$ is the distance from the point of muon decay in $\mathrm{cm}$, and $\mathrm{H}$ is the annual neutrino effective dose in $\mathrm{mSv}$. For consistency with the linear muon collider assumptions, $6.4 \times 10^{18}$ muon decays per year are assumed in subsequent calculations. Given the $\mathrm{TeV}$ muon energies and the earth shielding present, charged particle equilibrium is assumed to exist. Moreover, the neutrino beam is limited to muon neutrinos only.

The muon neutrino effective dose to fluence conversion factor is assumed to be valid at energies beyond those utilized in Cossairt et al. (1997). Given the TeV muon energies, Process D of Cossairt et al. (1997) will dominate the neutrino effective dose.

In deriving the circular muon collider effective dose relationship, a number of assumptions were made. First, the neutrino effective dose calculation assumes a $100 \%$ occupancy factor, and is an annual average based on the number of muon decays in a year. Second, the muon beam is well-collimated. In addition, the irradiated individual is (1) assumed to be within the footprint of the neutrino beam and the hadronic particle shower that results from the neutrino interactions, (2) irradiated by only one of the muon beam's decay neutrinos whose energy is one-half the total circular muon collider energy, and (3) uniformly irradiated by the neutrino and hadronic radiation types.

Table 5 summarizes the results of neutrino effective dose values as a function of distance from the muon decay location ( $r$ ) for a circular muon collider. Since the facility energy is the sum of the muon and antimuon energies, a $100 \mathrm{TeV}$ accelerator consists of a $50 \mathrm{TeV}$ muon beam and a $50 \mathrm{TeV}$ antimuon beam.

The long, thin conical radiation plumes present a radiation challenge well beyond the facility boundary. For example, a $25 \mathrm{TeV}$ circular muon collider produces a neutrino effective dose of $37 \mathrm{mSv} / \mathrm{y}$ at a distance of $1500 \mathrm{~km}$ from the facility. Although the neutrino effective dose plume will only have a radius of $12 \mathrm{~m}$ at $1500 \mathrm{~km}$, it presents a radiation challenge for muon collider health physicists and management. The effective dose values summarized in Table 5 have the potential to impart lethal doses to small areas. The large effective dose values and their control must be addressed in facility design and licensing.

The importance of properly characterizing offsite public effective doses is illustrated by the Fukushima Daiichi Nuclear Power Station (FDNPS) accident in Japan (Butler; 2011a, 2011b). These doses focused attention on inadequacies in the FDNPS design and licensing bases. Offsite effective doses and their profile must be carefully and credibly addressed in muon collider design and licensing evaluations. 


\begin{tabular}{|c|c|c|c|c|c|}
\hline Accelerator Energy $(\mathrm{TeV})^{\mathrm{a}}$ & \multicolumn{5}{|c|}{$\mathrm{H}(\mathrm{mSv} / \mathrm{y})$ at the Specified Distance $(\mathrm{r})$ from the Accelerator } \\
\cline { 2 - 6 } & $5 \mathrm{~km}$ & $25 \mathrm{~km}$ & $100 \mathrm{~km}$ & $1500 \mathrm{~km}$ & $2500 \mathrm{~km}$ \\
\hline 0.1 & $8.5 \times 10^{-4}$ & $3.4 \times 10^{-5}$ & $2.1 \times 10^{-6}$ & $9.4 \times 10^{-9}$ & $3.4 \times 10^{-9}$ \\
\hline 2 & 140 & 5.4 & 0.34 & $1.5 \times 10^{-3}$ & $5.4 \times 10^{-4}$ \\
\hline 25 & $3.3 \times 10^{6}$ & $1.3 \times 10^{5}$ & $8.3 \times 10^{3}$ & 37 & 13 \\
\hline 100 & $8.5 \times 10^{8}$ & $3.4 \times 10^{7}$ & $2.1 \times 10^{6}$ & $9.4 \times 10^{3}$ & $3.4 \times 10^{3}$ \\
\hline 500 & $5.3 \times 10^{11}$ & $2.1 \times 10^{10}$ & $1.3 \times 10^{9}$ & $5.9 \times 10^{6}$ & $2.1 \times 10^{6}$ \\
\hline 1000 & $8.5 \times 10^{12}$ & $3.4 \times 10^{11}$ & $2.1 \times 10^{10}$ & $9.4 \times 10^{7}$ & $3.4 \times 10^{7}$ \\
\hline
\end{tabular}

a The muon beam energy is half the accelerator energy.

Table 5. Annual Neutrino Effective Doses for a Circular Muon Collider.

Physics and cost parameters associated with $0.1,3,10$, and $100 \mathrm{TeV}$ circular muon colliders (King 1999a) are summarized in Table 6. Given current levels of technology, the collider cost will present a funding challenge as $\mathrm{TeV}$ muon energies are reached. In addition to funding issues, the control of radiation from the muon beams and neutrino plumes must be addressed. The feasibility of higher energy colliders will necessarily depend on technological development as well as financial support of scientific agencies.

\begin{tabular}{|c|c|c|c|c|}
\hline Accelerator Energy (TeV) & 0.1 & 3 & 10 & 100 \\
\hline Circumference (km) & 0.35 & 6 & 15 & 100 \\
\hline Average Magnetic Field (T) & 3.0 & 5.2 & 7.0 & 10.5 \\
\hline Cost & Feasible & Challenging & Challenging & Problematic \\
\hline
\end{tabular}

Table 6. Circular Muon Collider Physics and Cost Parameters.

As the collider energy increases, muon shielding requirements dictate a subsurface facility. The impact of locating the muon collider deeper underground with increasing accelerator energy can also be investigated. Using Eq. 28 and the data summarized in Table 4, permit the calculation of the neutrino effective dose upon its exit from the earth's surface. If the same beam properties are assumed as for the linear muon collider (i.e., $\mathrm{N}=6.4 \times 10^{18}$ muon decays per year) and $\mathrm{r}=\mathrm{L}$ (Table 4 ), then the magnitude and size of the resultant radiation plumes derived from Eq. 28 are summarized in Table 7.

\begin{tabular}{|c|c|c|c|c|}
\hline $\begin{array}{c}\text { Muon Energy } \\
(\mathrm{TeV})^{\mathrm{a}}\end{array}$ & $\begin{array}{c}\mathrm{d} \\
(\mathrm{m})^{\mathrm{b}}\end{array}$ & $\begin{array}{c}\text { L (Horizontal Distance at the } \\
\text { Earth's Surface) }(\mathrm{km})^{\mathrm{c}}\end{array}$ & $\begin{array}{c}\text { Beam Radius at the } \\
\text { Earth's Surface }(\mathrm{m})^{\mathrm{d}}\end{array}$ & $\begin{array}{c}\text { H at the Earth's } \\
\text { Surface } \\
(\mathrm{mSv} / \mathrm{y})\end{array}$ \\
\hline 1 & 100 & 36 & 3.6 & 2.6 \\
\hline 2 & 100 & 36 & 1.8 & 42 \\
\hline 5 & 200 & 51 & 1.0 & 820 \\
\hline 10 & 500 & 80.5 & 0.8 & $5.2 \times 10^{3}$ \\
\hline 50 & 500 & 80.5 & 0.16 & $3.3 \times 10^{6}$ \\
\hline 100 & 500 & 80.5 & 0.081 & $5.2 \times 10^{7}$ \\
\hline 500 & 500 & 80.5 & 0.016 & $3.3 \times 10^{10}$ \\
\hline 1000 & 500 & 80.5 & 0.0081 & $5.2 \times 10^{11}$ \\
\hline
\end{tabular}

a The accelerator energy is twice the muon energy.

${ }^{b}$ Accelerator depth below the surface of the earth.

${ }^{c}$ Horizontal exit point distance from the surface of the earth.

d The half-divergence angle is determined from Eq. 5.

Table 7. Neutrino Effective Dose Characteristics for a Circular Muon Collider. 
Although the effective dose results at the earth's surface are significant, they occur over a relatively small area. The results also assume a 100\% occupancy factor for this small area, which is not likely. The magnitude of the neutrino effective dose merits significant attention and emphasis on radiation monitoring and control. For example, a $500 \mathrm{TeV}$ muon beam would deliver an acute absorbed dose rate of about $1 \mathrm{~Gy} / \mathrm{s}$ to a $3.2 \mathrm{~cm}$ diameter circle. This absorbed dose rate is sufficient to deliver a biological detriment to the body within seconds (Bevelacqua, 2010a).

Dose management controls will be similar to those enacted for direct beam exposures at conventional accelerators. Interlocks associated with beam misalignment are effective in limiting the probability that the beam is directed toward an unanticipated direction. However, additional methods to control the offsite neutrino dose must be developed because lethal exposures can occur in a very short time even though the areas involved are small. Subjecting the public to potentially lethal effective doses represents unique facility licensing challenges that must be addressed in facility safety analyses. Public perception and stakeholder involvement will be key elements in licensing TeV-PeV scale muon colliders. The need for public involvement in licensing and regulatory discussions becomes particularly important when high effective doses could result from facility operations.

\section{Offsite effective dose considerations for muon colliders}

$\mathrm{TeV}$ energy neutrinos do not behave according to conventional operational health physics experience at power reactors and contemporary accelerator facilities. As noted previously, neutrinos are electrically uncharged and only interact through the weak interaction. Their small, but non-zero, interaction cross section creates a unique situation in terms of the behavior of the neutrino effective dose, particularly in terms of the shape and energy dependence of their radiation profile. These properties will lead to a modification of conventional health physics dose reduction concepts when applied to planned muon colliders.

Basic radiation protection principles suggest that the effective dose at a given location is reduced if the exposure time is minimized, the distance from the source is increased, or shielding is added between the source and the point of interest (Bevelacqua, 2009, 2010a). These principles must be modified at a TeV energy muon collider. The time principle is still valid for muons and neutrinos. The neutrino and muon effective doses are reduced by decreasing the exposure time.

The distance principle is ineffective when neutrinos are involved. Since neutrinos interact very weakly, relatively long distances are not effective in significantly reducing the neutrino effective dose. In fact, the neutrino beam remains a hazard for hundreds of kilometers. However, distance will still be effective for reducing the muon effective dose.

Unlike other radiation types, shielding neutrinos increases the effective dose. The magnitude of the particle showers produced by neutrino interactions is governed by the quantity of shielding material between the neutrino beam and the point of interest. However, shielding muons is an effective dose reduction measure.

From the standpoint of $\mathrm{TeV}$ energy neutrino radiation, a linear muon collider has a number of advantages over circular muon colliders. Firstly, the radiation is confined to two narrow 
beams that can be oriented to minimize the interaction of the neutrinos. A simple dose reduction technique orients the linear accelerators at an angle such that the neutrino beams exit the accelerator above the ground. This configuration minimizes the residual neutrino interactions with the earth and man-made structures. Secondly, the spent muons can be removed from the beam following collisions or interactions before they decay into highenergy neutrinos.

\section{Other radiation protection issues}

A number of radiation protection issues associated with TeV energy muon colliders will challenge accelerator health physicists. The issues related to large neutrino effective dose values and effective neutrino dosimetry were previously noted. Before construction of a muon collider, thorough studies will be performed to define the accelerator's radiation footprint. These studies will: (1) define muon collider shielding requirements; (2) assess induced activity within the facility and the environment (e.g., air, water, and soil), including the extent of groundwater activation; (3) assess radiation streaming through facility penetrations (e.g., ventilation ducts and access points); (4) assess various accident scenarios such as loss of power or beam misdirection; and (5) assess the various pathways for liquid and airborne releases of radioactive material. Facility waste generation and decommissioning are other areas that will require evaluation.

In addition to the aforementioned radiation protection issues, the $\mathrm{TeV}$ energy neutrino beam will create new issues. Radiation protection concerns unique to muon colliders have been reported by Autin et al. (1999), Bevelacqua (2004), Johnson et al. (1998), Mokhov \& Cossairt (1998), and Mokhov et al. (2000). These authors suggest that above about $1.5 \mathrm{TeV}$, the neutrino induced secondary radiation will pose a significant hazard even at distances on the order of tens to hundreds of kilometers. The neutrino radiation hazard presents both a physical as well as political challenge (King, 1999a).

These issues also complicate the process for locating a suitable site for a $\mathrm{TeV}$ energy muon collider. There are a number of potential solutions to reduce the neutrino effective dose associated with a muon collider. These include using radiation boundaries or fenced-off areas to denote areas with elevated effective dose values. Building the collider on elevated ground or at an isolated area would also minimize human exposure. Effective dose reduction measures are also available for specific muon collider configurations.

In a linear muon collider operating at the higher $\mathrm{TeV}$ energies, dose reduction is achieved by locating the interaction region above the earth's surface. In a circular muon collider, dose reduction is achieved by minimizing the straight sections in the ring, burying the collider deep underground to increase the distance before the neutrino beam exits the ground, and orienting the collider ring to take advantage of natural topographical features.

Orders of magnitude reductions in the neutrino effective dose are required for the muon colliders noted in this chapter (See Tables 3, 5, and 7) to meet current regulations for public exposures (ICRP, 2007). Some of the possible effective dose reduction solutions may be difficult to implement for the TeV energy muon colliders. The most feasible options for locating and operating the highest TeV energy muon collider are to either use (1) an isolated location where no one is exposed to the neutrino radiation before it exits into the atmosphere as a result of the earth's curvature, or (2) a linear muon collider 
constructed such that the individual muon beams collide in air well above the earth's surface.

For Option 1, the accelerator could either be constructed at an elevated location or at an isolated area. The area will need to be large, perhaps having a site boundary with a diameter greater than $100 \mathrm{~km}$ (King, 1999a). This size requirement restricts the available locations, and would normally require that the facility have access to the resources of an existing accelerator facility such as CERN or Fermilab. Alternatively, the facility could be located in an isolated area and scientific personnel relocated to that area with the establishment of a self-sufficient site. The final decision regarding facility location will involve funding and political considerations that are part of new facility development, licensing, and construction.

Option 2 would be technically feasible, and could be located at a smaller site. However, design considerations for both Options 1 and 2 would need to address a number of potential radiation issues associated with accelerator operation (Bevelacqua, 2008, 2009, and 2010a) that could lead to significant, unanticipated radiation levels in controlled as well as uncontrolled areas. Radiation protection issues include beam alignment errors, design errors, unauthorized changes, activation sources, and control of miscellaneous radiation sources (Bevelacqua, 2008, 2009, 2010a). These operational issues require close control because they have the potential to produce large and unanticipated effective dose values.

Beam alignment errors could direct the beam in unanticipated directions. Given the long range of the muon effective dose profile, these errors could have a significant impact on licensing and accident analysis. Beam alignment errors are caused by a variety of factors including power failures, maintenance errors, and magnet failures. Both human errors and mechanical failures lead to beam alignment issues.

Changes in the beam energy or beam current, that exceed the authorized operating envelope, lead to elevated fluence rates, the creation of unanticipated particles, or the creation of particles with higher energy than anticipated. Changes to beam parameters must be carefully evaluated for their impact on the radiation environment of the facility.

The control of secondary radiation sources, radio-frequency equipment, high-voltage power supplies, and other experimental equipment merits special attention. These sources of radiation are more difficult to control than the primary or scattered accelerator radiation because health physicists may not be aware of their existence, the experimenters may not be aware of the hazard, or the radiation source is at least partially masked by the accelerator's radiation output. These miscellaneous radiation sources will include $\mathrm{x}$-rays as well as other types of radiation.

\section{Overview of the neutrino effective dose at a tau collider}

A third generation tau collider has not been evaluated. In order to provide an estimate of the effective dose consequences of a tau collider, a modification of the muon collider methodology is utilized. The decay characteristics of a tau are considerably more complex than muon decay. The muon essentially decays with a branching ratio of $100 \%$ into a lepton and neutrinos via Eq. 1. For example, tau decays involve 119 decay modes with specified branching fractions with six modes accounting for $90 \%$ of the decays (Particle Data Group 2010). The dominant tau decay mode is: 


$$
\tau^{-} \rightarrow \pi^{-}+\pi^{0}+v_{\tau}(25.51 \%)
$$

However, the negative pion dominantly decays into a muon and antimuon neutrino, and the neutral pion decays primarily into photons.

$$
\tau^{-} \rightarrow\left(\mu^{-}+\bar{v}_{\mu}\right)+(\gamma+\gamma)+v_{\tau}
$$

Subsequently, the muon decays following Eq. 1. Eq. 30 then yields:

$$
\tau^{-} \rightarrow\left(e^{-}+v_{\mu}+\bar{v}_{e}+\bar{v}_{\mu}\right)+(\gamma+\gamma)+v_{\tau}
$$

The net result of the decay is that multiple neutrinos are produced from the tau and subsequent decay of particles. The factor $\xi$ described in subsequent discussion incorporates the effects of the multiple tau decay modes and their effects on the neutrino effective dose.

Subsequent discussion assumes no annihilation of particles and antiparticles in the beam produced by the tau decay products. In addition, the narrow beam approximation is assumed.

The neutrino dose from tau decays is determined by comparing the number of neutrinos emitted from an equal number of tau and muon decays. $\xi$ defines the ratio of the number of neutrinos contributing to the tau collider to muon collider effective doses:

$$
\xi=\frac{\sum_{i=1}^{N} Y_{i} \sum_{j=1}^{3}\left(a n_{i}\left(v_{j}\right)+b n_{i}\left(\bar{v}_{j}\right)\right)}{a n\left(v_{\mu}\right)+b n\left(\bar{v}_{e}\right)}
$$

In the numerator of Eq. 32, i labels the various decay modes of the tau, $\mathrm{N}$ is the number of tau decay modes, $Y_{i}$ is the branching fraction of the ith tau decay mode, $n_{i}\left(v_{j}\right)$ is the number of generation $\mathrm{j}$ neutrinos emitted from decay mode $\mathrm{i}$, and $n_{i}\left(\bar{v}_{j}\right)$ is the number of generation $\mathrm{j}$ antineutrinos emitted from decay mode i. In the denominator of Eq. (32), $\mathrm{n}\left(\mathrm{v}_{\mu}\right)$ is the number of muon neutrinos emitted in a muon decay, and $n\left(\bar{v}_{e}\right)$ is the number of antielectron neutrinos emitted in a muon decay. The $\mathrm{j}$ sum counts the three neutrino generations, and $a$ and $b$ are the cross-section factors of King (1999a) for neutrinos and antineutrinos which are 1.0 and 0.5 , respectively.

The ratio of tau neutrino to muon neutrino effective doses is obtained by utilizing the value of $\xi$ and the calculated ratio of tau and muon neutrino cross-sections ( $\beta$ ) (Jeong \& Reno, 2010). The discussion is applicable to circular and linear muon and tau colliders. For equivalent accelerator operating conditions (e.g., beam energy and number of beam particle decays) and receptor conditions (e.g., distance and ambient conditions), the ratio of neutrino effective doses from a tau collider and muon collider is given by:

$$
\frac{H_{\tau^{-}}(E)}{H_{\mu^{-}}(E)}=\xi \beta(E)
$$


The results of calculations utilizing Eq. 33 are summarized in Table 8.

\begin{tabular}{|c|c|}
\hline Beam Energy (TeV) & Effective Dose Ratio \\
\hline 0.01 & 0.39 \\
\hline 0.1 & 1.75 \\
\hline 1.0 & 2.16 \\
\hline 10. & 2.23 \\
\hline
\end{tabular}

Table 8. Ratio of Tau and Muon Collider Neutrino Effective Doses.

The tau collider neutrino effective doses are generally larger than those encountered in a muon collider, and the tau dose profile is also larger. The larger tau profile is demonstrated by considering Eqs. 3 and 4 for equivalent tau and muon collider configurations:

$$
\frac{r\left(\tau^{-}\right)}{r\left(\mu^{-}\right)}=\frac{m_{\tau^{-}}}{m_{\mu^{-}}}=\frac{1777 \mathrm{MeV}}{105.7 \mathrm{MeV}}=16.8
$$

Using Eq. 34 and the Table 7 results for circular tau collider conditions, the neutrino effective dose profile radius at the earth's surface is $60.5,30.2,16.8$, and $13.4 \mathrm{~m}$ for $1,2,5$, and $10 \mathrm{TeV}$ beams. These affected areas and associated effective doses suggest that the tau collider is a more significant radiation hazard than the muon collider. Therefore, larger effective doses and affected areas are anticipated during tau collider operations.

An improved calculation of the neutrino effective dose from a tau collider requires a better specification of neutrino properties. For example, previous calculations were based on the Standard Model assumption that neutrinos have zero mass. Neutrino masses can be calculated assuming the alternative gauge group $S U(2)_{L} \otimes S U(2)_{R} \otimes U(1)$ instead of the Standard Model $S U(2)_{L} \otimes U(1)$. This gauge group leads to a neutrino generation i mass:

$$
m_{i}=\frac{M_{i}^{2}}{g m_{W_{R}}}
$$

where $M_{i}$ is the generation i lepton mass $(e, \mu$, and $\tau), W_{R}$ is the right-handed $W$ boson mass $(\geq 300 \mathrm{GeV}$ ), and $\mathrm{g}$ is a coupling constant with a value of 0.585 (Mohapatra \& Senjanović, 1980). Using these values in Eq. 35 leads to electron, muon, and tau neutrino upper bound masses of $1.5 \mathrm{eV}, 64 \mathrm{keV}$, and $18 \mathrm{MeV}$, respectively. These masses affect the input values used to calculate the neutrino effective dose in Eqs. 14 and 23. As an alternative, better crosssection data and dose conversion factors would refine the neutrino effective dose.

\section{Conclusions}

Neutrino radiation will be a health physics issue and design constraint for muon colliders, particularly at $\mathrm{TeV}$ energies. $\mathrm{TeV}$ energy muon colliders will require careful site selection and the neutrino effective dose may dictate that these machines be constructed in isolated areas. With the operation of $\mathrm{TeV}$ energy muon colliders, the neutrino effective dose can no longer be neglected. Neutrino detection, neutrino dosimetry, and the determination of the neutrino effective dose will no longer be academic exercises, but will become operational 
health physics concerns. Keeping public and occupational neutrino effective doses below regulatory limits will require careful and consistent application of dose reduction methods.

When compared to muon colliders, initial scooping calculations for tau colliders suggest that higher effective doses and affected areas will result from their operation. Although, the tau collider calculations are initial estimates, they suggest that significant radiation challenges are also presented by these machines.

\section{References}

Autin, B; Blondel, A. \& Ellis, J. (1999). Prospective Study of Muon Storage Rings at CERN, CERN 99-02, European Laboratory for Particle Physics, Geneva, Switzerland

Bevelacqua, J. (2004). Muon Colliders and Neutrino Dose Equivalents: ALARA Challenges for the 21st Century, Radiation Protection Management, Vol.21, No. 4, pp. 8-30.

Bevelacqua, J. (2008). Health Physics in the 21st Century, Wiley-VCH, ISBN 9783527408221, Weinheim, Germany

Bevelacqua, J. (2009). Contemporary Health Physics: Problems and Solutions (Second Edition), ISBN 9783527408245, Weinheim, Germany

Bevelacqua, J. (2010a). Basic Health Physics: Problems and Solutions (Second Edition), ISBN 9783527408238, Weinheim, Germany

Bevelacqua, J. (2010b). Standard Model of Particle Physics-A Health Physics Perspective, Health Physics, Vol.99, No.5, pp. 613-623

Butler, D. (2011a). Radioactivity Spreads in Japan, Nature, Vol.471, No.7340, pp. 555-556

Butler, D. (2011b). Fukushima Health Risks Scrutinized, Nature, Vol.472, No.7341, pp. 13-14

Cottingham, W. \& Greenwood, D. (2007). An Introduction to the Standard Model of Particle Physics (Second Edition), Cambridge University Press, ISBN 9780521852494, Cambridge, UK

Cossairt, J.; Grossman, N. \& Marshall, E. (1996). Neutrino Radiation Hazards: A Paper Tiger, Fermilab-Conf-96/324, Accessed on July 11, 2011, Available from: <http://lss.fnal.gov/archive/1996/conf/Conf-96-324.pdf>

Cossairt, J.; Grossman, N. \& Marshall, E. (1997). Assessment of Dose Equivalent due to Neutrinos, Health Physics, Vol.73, No.6, 894-898.

Cossairt, J. \& Marshall, E. (1997). Comment on "Biological Effects of Stellar Collapse Neutrinos, Physical Review Letters, Vol.78, No.7, pp.1394.

Collar, J. (1996). Biological Effects of Stellar Collapse Neutrinos, Physical Review Letters, Vol.76, No.6, pp. 999-1002

Geer, S. (2010). From Neutrino Factory to Muon Collider, FERMILAB-CONF-10-024-APC, Accessed on July 14, 2011, Available from: < http://arxiv.org/abs/1006.0923>

Griffiths, D. (2008). Introduction to Elementary Particle Physics (Second Edition), Wiley-VCH, ISBN 9783527406012, Weinheim, Germany

ICRP Report No. 60. (1991). 1990 Recommendations of the International Commission on Radiological Protection, Elsevier, Amsterdam

ICRP Report No. 107. (2007). The 2007 Recommendations of the International Commission on Radiological Protection, Elsevier, Amsterdam

Jeong, Y. \& Reno, M. (2010). Tau neutrino and antineutrino cross sections, Accessed on July 12, 2011, Available from:

<http://arxiv.org/PS_cache/arxiv/pdf/1007/1007.1966v1.pdf>

Johnson, C.; Rolandi, G. \& Silari, M. (1998). Radiological Hazard due to Neutrinos from a Muon Collider, Internal Report CERN/TIS-RP/IR/98, European Laboratory for 
Particle Physics (CERN), Geneva, Switzerland (1998). Accessed on July 12, 2011, Available from:

<http://www.physics.princeton.edu/mumu/johnson/neutrino.pdf>

King, B. (1999a). Studies for Muon Collider Parameters at Center-of-Mass Energies of $10 \mathrm{TeV}$ and $100 \mathrm{TeV}$, Brookhaven National Laboratory, Accessed on July 25, 2011, Available from: <http://arxiv.org/PS_cache/physics/pdf/9908/9908018v1.pdf >

King, B. (1999b). Neutrino Radiation Challenges and Proposed Solutions for Many-TeV Muon Colliders, Proc. HEMC'99 Workshop - Studies on Colliders and Collider Physics at the Highest Energies: Muon Colliders at $10 \mathrm{TeV}$ to $100 \mathrm{TeV}$, Montauk, NY, September 1999 Accessed on July 25, 2011, Available from: <http://nfmccdocdb.fnal.gov/cgibin/RetrieveFile?docid=119\&version=1\&filename=muc0119.ps.gz>

King, B. (1999c). Neutrino Physics at Muon Colliders, Brookhaven National Laboratory, Accessed on July 19, 2011, Available from: <http://arxiv.org/PS_cache/hepex/pdf/9907/9907035v1.pdf>

Kuno, Y. (2009). Project X Workshop Summary, Muon Collider Physics Workshop, Fermi National Laboratory, Batavia, IL, 10 -12 November, 2009, Available from: $<$ https:/ / indico.fnal.gov / getFile.py/access?contribId=78\&sessionId=0\&resId=0\& materialId=slides\&confId=2855>

Mohapatra, R. \& Senjanović, G. (1980). Neutrino Mass and Spontaneous Parity Nonconservation, Physical Review Letters, Vol.44, No.14, pp. 912-915

Mokhov, N. \& Cossairt, J. (1998). Radiation Studies at Fermilab, Proceedings of the Fourth Workshop on Simulating Accelerator Radiation Environments (SARE4), Knoxville, TN, 14 - 16 September, 1998, Accessed on July 25, 2011, Available from: <http://lss.fnal.gov/archive/1998/conf/Conf-98-384.pdf >

Mokhov, N.; Striganov, S. \& van Ginneken, A. (2000). Muons and Neutrinos at High Energy Accelerators, FERMILAB-Conf-00/182, Accessed on July 11, 2011, Available from: <http://lss.fnal.gov/archive/2000/conf/Conf-00-182.pdf>

NCRP Report No. 144. (2003). Radiation Protection for Particle Accelerator Facilities, National Council on Radiation Protection and Measurements, ISBN 0929600770, Bethesda, MD

Particle Data Group. (2010). Review of Particle Properties, Journal of Physics G, Vol.37, No.7A, pp. 1-1422.

Quigg, C. (1997). Neutrino Interaction Cross Sections, FERMILAB-Conf-97/158-T, Accessed on July 11, 2011, Available from: < http://lss.fnal.gov/archive/1997/conf/Conf97-158-T.pdf>

Silari, M. \& Vincke, H. (2002). Neutrino Radiation Hazard at the Planned CERN Neutrino Factory, Technical Note TIS-RP/TN/2002-01, Accessed on July 12, 2011, Available from: <http:/ / slap.web.cern.ch/slap/NuFact/NuFact/nf105.pdf>

Zimmerman, F. (1999). Final Focus Challenges for Muon Colliders at Highest Energies, Proc. HEMC'99 Workshop - Studies on Colliders and Collider Physics at the Highest Energies: Muon Colliders at $10 \mathrm{TeV}$ to $100 \mathrm{TeV}$, Montauk, NY, September 1999, Accessed on July 25, 2011, Available from: <//cdsweb.cern.ch/record/420774/files/sl-1999077.ps.gz>

Zisman, M. (2011). R\&D Toward a Neutrino Factory and Muon Collider, LBNL-4494E, Lawrence Berkeley National Laboratory, Berkeley, CA, Accessed on July 25, 2011, Available from: <http://escholarship.org/uc/item/43p7z0v1> 


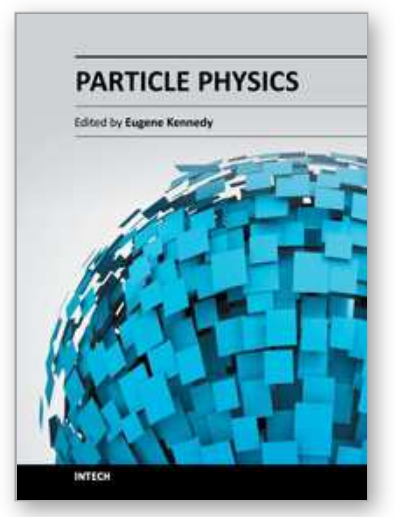

\author{
Particle Physics \\ Edited by Dr. Eugene Kennedy
}

ISBN 978-953-51-0481-0

Hard cover, 114 pages

Publisher InTech

Published online 20, April, 2012

Published in print edition April, 2012

Stimulated by the Large Hadron Collider and the search for the elusive Higgs Boson, interest in particle physics continues at a high level among scientists and the general public. This book includes theoretical aspects, with chapters outlining the generation model and a charged Higgs boson model as alternative scenarios to the Standard Model. An introduction is provided to postulated axion photon interactions and associated photon dispersion in magnetized media. The complexity of particle physics research requiring the synergistic combination of theory, hardware and computation is described in terms of the e-science paradigm. The book concludes with a chapter tackling potential radiation hazards associated with extremely weakly interacting neutrinos if produced in copious amounts with future high-energy muon-collider facilities.

\title{
How to reference
}

In order to correctly reference this scholarly work, feel free to copy and paste the following:

Joseph John Bevelacqua (2012). Muon Colliders and Neutrino Effective Doses, Particle Physics, Dr. Eugene Kennedy (Ed.), ISBN: 978-953-51-0481-0, InTech, Available from: http://www.intechopen.com/books/particlephysics/muon-colliders-and-neutrino-effective-doses

\section{INTECH}

open science | open minds

\section{InTech Europe}

University Campus STeP Ri

Slavka Krautzeka 83/A

51000 Rijeka, Croatia

Phone: +385 (51) 770447

Fax: +385 (51) 686166

www.intechopen.com

\section{InTech China}

Unit 405, Office Block, Hotel Equatorial Shanghai

No.65, Yan An Road (West), Shanghai, 200040, China

中国上海市延安西路65号上海国际贵都大饭店办公楼 405 单元

Phone: +86-21-62489820

Fax: $+86-21-62489821$ 
(C) 2012 The Author(s). Licensee IntechOpen. This is an open access article distributed under the terms of the Creative Commons Attribution 3.0 License, which permits unrestricted use, distribution, and reproduction in any medium, provided the original work is properly cited. 